\title{
The importance of scattering, surface potential, and vanguard counter-potential in terahertz emission from gallium arsenide
}

Cite as: Appl. Phys. Lett. 100, 261601 (2012); https://doi.org/10.1063/1.4730954

Submitted: 27 April 2012 . Accepted: 09 June 2012 . Published Online: 25 June 2012

D. L. Cortie, and R. A. Lewis
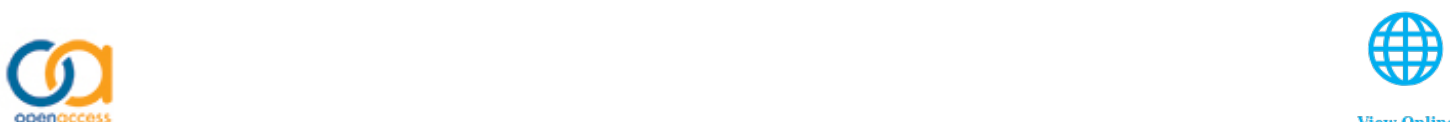

\section{ARTICLES YOU MAY BE INTERESTED IN}

Semiconducting and other major properties of gallium arsenide

Journal of Applied Physics 53, R123 (1982); https://doi.org/10.1063/1.331665

Optoelectronic measurement of semiconductor surfaces and interfaces with femtosecond optics

Journal of Applied Physics 71, 326 (1992); https://doi.org/10.1063/1.350710

Perspective: Terahertz science and technology

Journal of Applied Physics 122, 230901 (2017); https://doi.org/10.1063/1.5007683

\section{Lock-in Amplifiers up to $600 \mathrm{MHz}$}
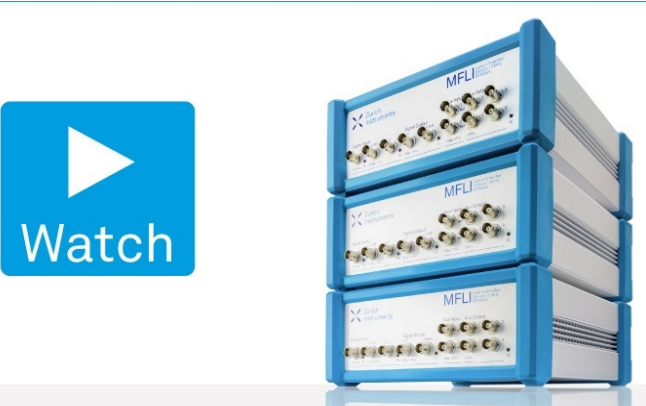


\title{
The importance of scattering, surface potential, and vanguard counter-potential in terahertz emission from gallium arsenide
}

\author{
D. L. Cortie ${ }^{\text {a) }}$ and R. A. Lewis ${ }^{\text {b) }}$ \\ Institute for Superconducting and Electronic Materials, University of Wollongong, Wollongong, \\ New South Wales 2522, Australia
}

(Received 27 April 2012; accepted 9 June 2012; published online 25 June 2012)

\begin{abstract}
It is well established that under excitation by short $(<1 \mathrm{ps})$, above-band-gap optical pulses, semiconductor surfaces may emit terahertz-frequency electromagnetic radiation via photocarrier diffusion (the dominant mechanism in InAs) or photocarrier drift (dominant in GaAs). Our three-dimensional ensemble Monte Carlo simulations allow multiple physical parameters to vary over wide ranges and provide unique direct insight into the factors controlling terahertz emission. We find for GaAs (in contrast to InAs), scattering and the surface potential are key factors. We further delineate in GaAs (as in InAs) the role of a vanguard counter-potential. The effects of varying dielectric constant, band-gap, and effective mass are similar in both emitter types. (C) 2012 American Institute of Physics. [http://dx.doi.org/10.1063/1.4730954]
\end{abstract}

The generation, detection, and utilization of terahertzfrequency electromagnetic radiation has burgeoned this last decade, as seen in the publication of recent reviews ${ }^{1-3}$ and books. ${ }^{4-9}$ A key development has been time-domain spectroscopy, which relies on the coherent detection of ultrashort pulses of terahertz radiation. ${ }^{10}$ This in turn has spurred a renewed interest in the mechanisms by which terahertz pulses are produced. Transient current flow at a semiconductor surface is one principal mechanism. ${ }^{11-14}$

Critical insights into terahertz radiation from semiconductor surfaces come from Monte Carlo simulations. ${ }^{15-26}$ Johnston et al. established that in-built surface depletion field and photo-Dember emitters may be distinguished, ${ }^{15}$ that magnetic fields increase emission by re-orientating rather than strengthening the radiating dipole, ${ }^{15,16}$ and that power-limiting mechanisms in photoconductive switches depend on pump pulse length. ${ }^{17}$ Malevich highlighted the roles of the cold and hot carriers ${ }^{18}$ and of the pump photon energy. ${ }^{19}$ Liu and co-workers reported the effect of large electric fields, ${ }^{20}$ the contrasting behaviors of $\mathrm{InSb}$ and InAs, ${ }^{21}$ as well as the dependence of emission on pump photon energy. ${ }^{22}$ Reklaitis modeled emission from GaAs $p-i-n$ structures $^{23}$ and bulk InAs (Ref. 25), proposed $\delta$-doped heterostructures as superior emitters, ${ }^{24}$ and found that the crossover from surface field to photo-Dember emission depends on pump photon energy. ${ }^{26}$

The previous work attempts in the main to reproduce extant experimental results. Here we extend the project. To determine the path to better emitters, we vary parameters in ways experimentally difficult. We first investigated the effect of many parameters on emission from InAs. ${ }^{27}$ We now turn from that diffusion-dominated narrow-gap case to the drift-dominated wide-gap case of GaAs to determine which physical parameters most strongly influence the emission of terahertz radiation.

\footnotetext{
${ }^{a)}$ Present address: The Bragg Institute, Australian Nuclear Science and Technology Organisation, Lucas Heights, New South Wales 2234, Australia. b)Electronic mail: roger@uow.edu.au. URL: http://uow.edu.au/ roger.
}

Our method has been set out in detail previously. ${ }^{27}$ It follows the usual, well-documented Monte Carlo approach $^{28-30}$ as applied to the problem of terahertz generation at semiconductor surfaces. ${ }^{15-21}$ The GaAs materials parameters we adopt are set out in Table I. Our calculated scattering rates for several scattering mechanisms are given in Fig. 1. As for InAs (see Fig. 1 of Ref. 27), polar optical phonon scattering is the main scattering mechanism in the relevant energy range for surface-accelerated electrons. The effect of scattering on the terahertz emission is given in Fig. 2. It is seen that the inclusion of optical polar scattering has a dramatic effect on the terahertz emission. The terahertz field is reduced to approximately two-thirds of its value without scattering. This corresponds to the terahertz amplitude being approximately half that of the fictional idealized case of no scattering. In this respect, GaAs differs significantly from InAs, in which transport may be considered to be almost collisionless on ps timescales. ${ }^{27}$ However, as for InAs, the additional mechanisms of carrier-carrier, intervalley, and impurity scattering play little role (Fig. 2). So means to reduce optical polar scattering, such as lower temperature,

TABLE I. GaAs physical parameters used in the model. These are identical to Ref. 15, with the speed of sound added.

\begin{tabular}{ll}
\hline \hline Parameter & Value \\
\hline Doping density, $n=n_{i}=p_{i}\left(\mathrm{~m}^{-3}\right)$ & $2 \times 10^{21}$ \\
Bandgap, $E_{g}(\mathrm{eV})$ & 1.5 \\
Low-frequency dielectric constant, $\epsilon(0) / \epsilon_{0}$ & 12.95 \\
High-frequency dielectric constant, $\epsilon(\infty) / \epsilon_{0}$ & 10.9 \\
Effective electron mass in $\Gamma$ valley, $m_{e} / m_{0}$ & 0.067 \\
Effective hole mass, $m_{h} / m_{0}$ & 0.5 \\
Effective electron mass in $L$ valley, $m_{e} / m_{0}$ & 0.35 \\
$L-\Gamma$ valley energy offset, $E_{L-\Gamma}(\mathrm{eV})$ & 0.29 \\
Mass density, $\left.\rho(\mathrm{kg} \mathrm{m})^{-3}\right)$ & 5360 \\
Photon absorption coefficient, $\alpha\left(\mathrm{m}^{-1}\right)$ & $1.2 \times 10^{6}$ \\
LO phonon energy, $\hbar \omega_{\mathrm{LO}}(\mathrm{eV})$ & 0.035 \\
Speed of sound, $(100)$ direction, $v_{s}(\mathrm{~m} / \mathrm{s})$ & 4739 \\
Deformation potential, $\mathrm{D}_{L \Gamma}=D_{t} K(\mathrm{eV} / \mathrm{m})$ & $0.6 \times 10^{10}$ \\
Laser photon energy, $E_{\mathrm{photon}}(\mathrm{eV})$ & 1.55 \\
\hline \hline
\end{tabular}




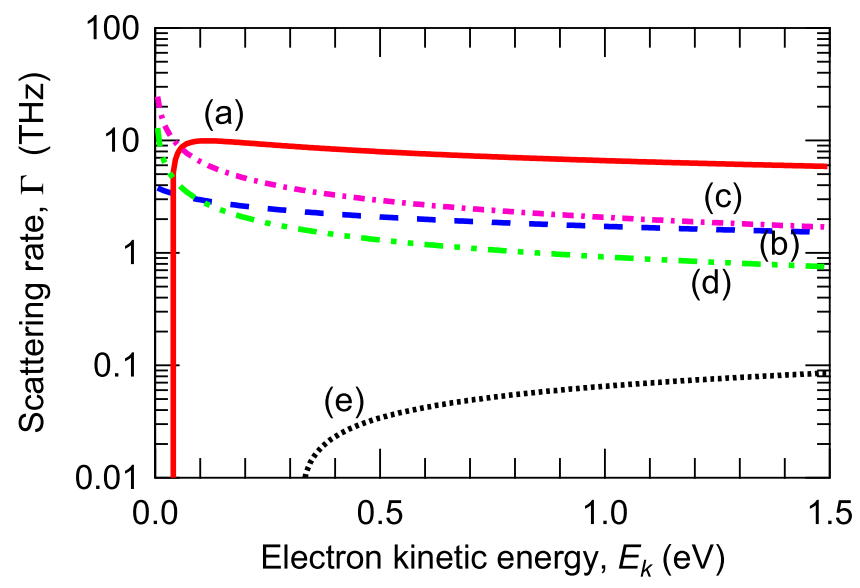

FIG. 1. Calculated electron scattering rates in GaAs as a function of electron kinetic energy. (a) Polar optical phonon emission. (b) Polar optical phonon absorption. (c) Upper limit for carrier-carrier scattering. (d) Ionizedimpurity scattering. (e) Intervalley scattering.

would be efficacious in GaAs terahertz emitters but not in InAs. The vertical scale of Fig. 2 (and of all subsequent figures) is normalized to the case of InAs under identical excitation. The terahertz field is approximately ten times less from GaAs (and so the field amplitude approximately 100 times less) than from InAs. The width is about double and so the terahertz bandwidth about half.

The effect of varying the dark surface potential is given in Fig. 3. A large surface potential of either polarity results in a large terahertz field; the polarity of the field reflects the polarity of the potential, as the dominant mechanism of charge carrier transport is drift induced by the surface field. Even so, the effect of diffusion (the photo-Dember effect) cannot be dismissed entirely. It may be noted that the minimum in terahertz field does not occur at exactly zero surface potential but at approximately $+0.2 \mathrm{~V}$. It may also be noted that at positive surface potentials, for example, at $+1 \mathrm{~V}$, the terahertz field is less than at the corresponding negative surface potential, in this case $-1 \mathrm{~V}$. This asymmetry is related

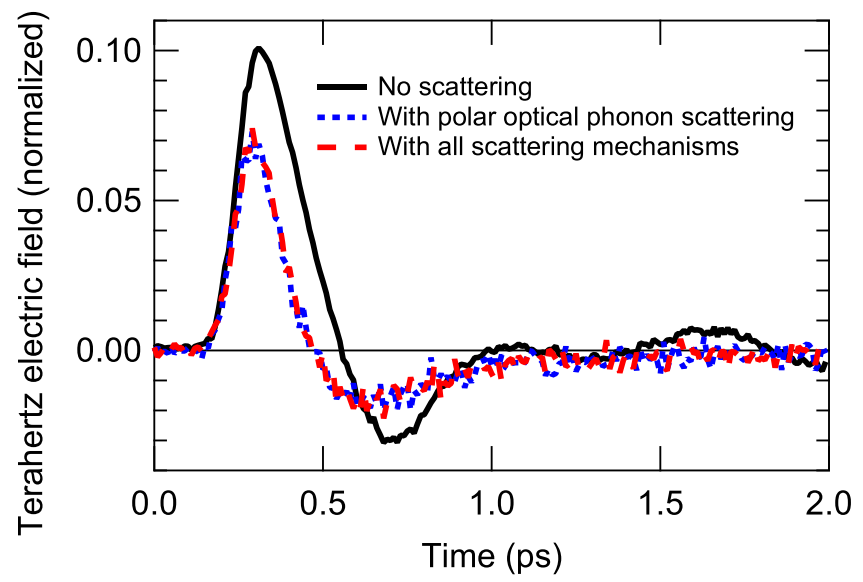

FIG. 2. The major role played by polar optical phonon scattering in reducing terahertz emission from GaAs. The full curve represents the electrodynamic simulation without scattering. The dotted curve includes only polar optical phonon scattering. This reduces the peak terahertz field by approximately one third. Little further change results when carrier-carrier, intervalley, and ionized-impurity scattering are included in the simulation, represented by the dashed curve.

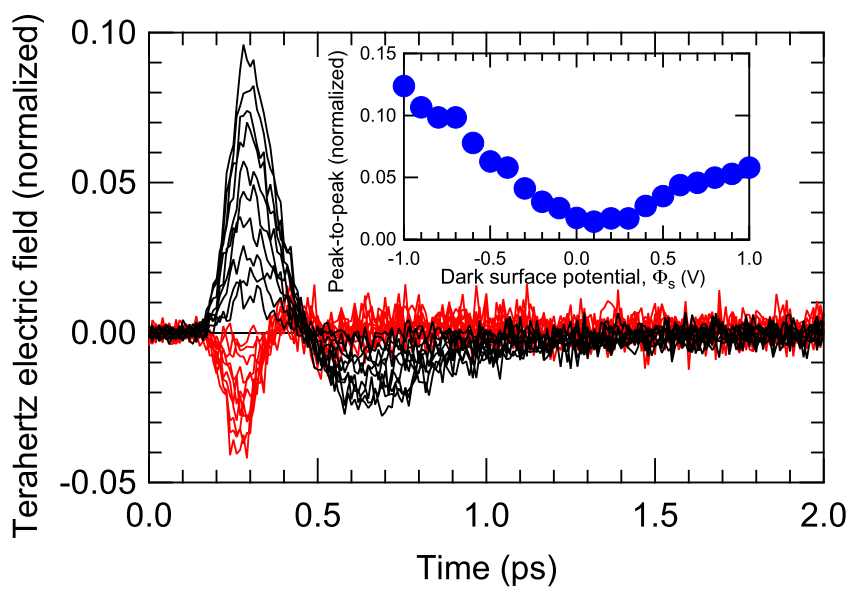

FIG. 3. The effect of surface potential on terahertz emission from GaAs. Red (black) traces correspond to positive (negative) potentials. A positive (negative) potential attracts (repels) electrons to (from) the surface to form an electron accumulation (depletion) layer, or downward (upward) conduction band bending. At large potentials of opposite polarities, the sign of the terahertz field is reversed. There is a slight asymmetry; negative potentials assist the photo-Dember effect whereas positive potentials compete with it.

to the interplay between the drift and diffusion mechanisms. For negative surface potentials, the two effects add, and a stronger emission results. For positive surface potentials, the diffusion mechanism is in competition with the drift mechanism, and a weaker emission results. These effects are in stark contrast to the case of InAs, where varying the surface potential over the same range has very little effect on terahertz output (see Fig. 5 of Ref. 27), as might be expected for a narrow-gap semiconductor.

The role of the pump laser pulse length is given in Fig. 4. As for InAs, reducing the pulse width increases the emission. (In contrast, reducing the pulse width below 40 fs in GaAs photoconductive switches was found to diminish the terahertz emission. ${ }^{17}$ ) Due to the smaller absorption coefficient, the effect of the vanguard counter-potential is not as strong as in InAs. ${ }^{27}$ The Dember effect is reduced, so a lower proportion of electrons escape the surface region to form the counter-potential on these time scales. Artificially increasing the absorption coefficient (inset to Fig. 4) shows an enhancement, as is expected.

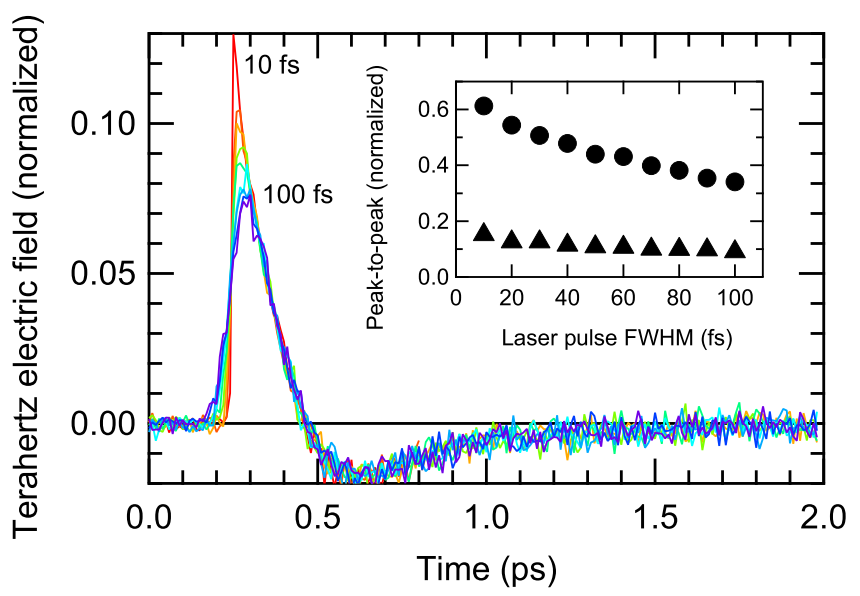

FIG. 4. Role of laser pulse length on terahertz radiation from GaAs. (Inset) The peak-to-peak intensity for usual photon absorption coefficient $\left(1.2 \times 10^{6} \mathrm{~m}^{-1}\right.$; triangles $)$ and a large absorption coefficient $\left(10 \times 10^{6} \mathrm{~m}^{-1}\right.$; circles). 


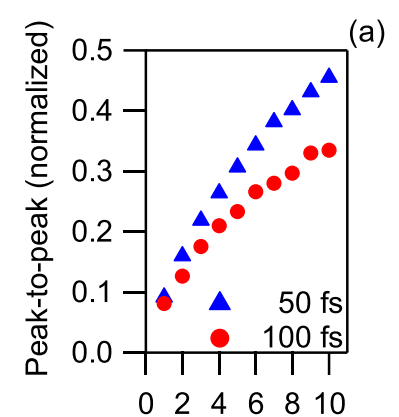

Absorption coefficient $\left(\times 10^{6} \mathrm{~m}^{-1}\right)$

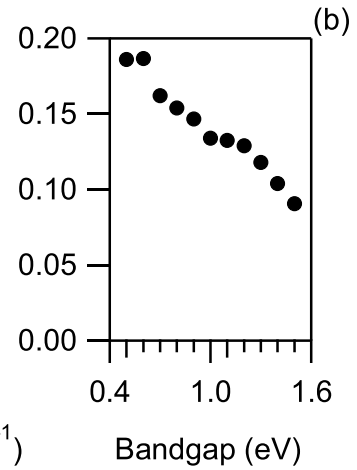

(b)

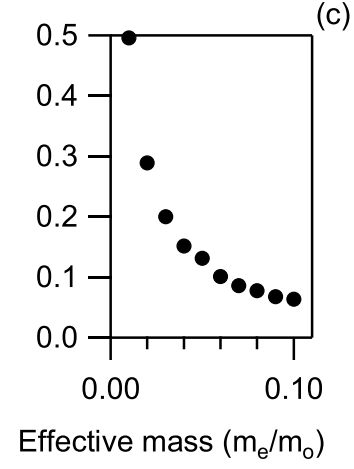

(c)

FIG. 5. Role of (a) absorption coefficient, (b) bandgap, and (c) effective mass on surface terahertz emission.
In practice, it is not simple to systematically vary the absorption coefficient, bandgap, or the effective mass, but we have calculated these effects (Fig. 5). We find as the absorption coefficient increases, so does the terahertz emission, as expected. The effect begins to saturate for longer pulses, a consequence of the vanguard counter-potential. As the bandgap is reduced, terahertz emission is increased. This is a consequence of more energy being available from the pump photons after producing the photoelectron-hole pair and this energy excess is taken up in the photocarrier motion. As the effective mass is reduced, the terahertz emission is increased. This is directly related to the increase in differential carrier mobility.

In summary, our study has identified the distinctive character of terahertz emission from GaAs, which is delineated against the background InAs, the subject of previous study. ${ }^{27}$ First, the terahertz field generated from GaAs is an order of magnitude less than from InAs. Second, it is strongly reduced by polar optical phonon scattering, which is not the case with InAs (Fig. 2). Improved performance from GaAs could be realized by reducing this main scattering source, for example, by cooling the GaAs, while less advantage would be gained in InAs. Third, in direct contrast to InAs, the surface field plays a central role in the terahertz emission from GaAs (Fig. 3). Increasing the surface field greatly assists terahertz emission, more so if the potential is negative than positive, as then the potential works with, rather than against, the photo-Dember effect. Hence, surface field engineering has greater scope to improve further the emission from GaAs than from InAs. Fourth, the vanguard counter-potential, though present, is weaker than in the case of InAs. Hence the advantage of using ultrashort pump pulses is not so great for GaAs. It follows that simpler and less expensive sources of longer laser pulses, for example, fiber lasers rather than Ti:sapphire lasers, are relatively better suited to pumping GaAs than InAs.

This work was supported by the Australian Research Council, the University of Wollongong, and the Chinese
Academy of Sciences Visiting Professorship for Senior International Scientists Grant \#2010T2J07.

${ }^{1}$ P. H. Siegel, IEEE Trans. Microwave Theory Tech. 50, 910 (2002).

${ }^{2}$ B. Ferguson and X.-C. Zhang, Nature Mater. 1, 26 (2002).

${ }^{3}$ R. A. Lewis, Proc. IEEE 95, 1641 (2007).

${ }^{4}$ Sensing with Terahertz Radiation, edited by D. Mittleman (Springer, Berlin, 2003).

${ }^{5}$ Terahertz Optoelectronics, edited by K. Sakai (Springer, Berlin, 2005).

${ }^{6}$ Terahertz Spectroscopy: Principles and Applications, edited by S. L. Dexheimer (CRC, Boca Raton, 2007).

${ }^{7}$ S. D. Ganichev and W. Prettl, Intense Terahertz Excitation of Semiconductors (Oxford University Press, Oxford, 2005).

${ }^{8}$ Y.-S. Lee, Principles of Terahertz Science and Technology (Springer, Berlin, 2009).

${ }^{9}$ X.-C. Zhang and J. Xu, Introduction to THz Wave Photonics (Springer, New York, 2010).

${ }^{10}$ C. A. Schmuttenmaer, Chem. Rev. 104, 1759 (2004).

${ }^{11}$ X. C. Zhang, B. B. Hu, J. T. Darrow, and D. H. Auston, Appl. Phys. Lett. 56, 1011 (1990).

${ }^{12}$ J. Lloyd-Hughes, E. Castro-Camus, M. D. Fraser, C. Jagadish, and M. B. Johnston, Phys. Rev. B 70, 235330 (2004).

${ }^{13}$ V. L. Malevich, R. Adomavičius, and A. Krotkus, C. R. Phys. 9, 130 (2008).

${ }^{14}$ R. Mendis, M. L. Smith, L. J. Bignell, R. E. M. Vickers, and R. A. Lewis, J. Appl. Phys. 98, 126104 (2005).

${ }^{15}$ M. B. Johnston, D. M. Whittaker, A. Corchia, A. G. Davies, and E. H. Linfield, Phys. Rev. B 65, 165301 (2002).

${ }^{16}$ M. B. Johnston, D. M. Whittaker, A. Corchia, A. G. Davies, and E. H. Linfield, J. Appl. Phys. 91, 2104 (2002).

${ }^{17}$ E. Castro-Camus, J. Lloyd-Hughes, and M. B. Johnston, Phys. Rev. B 71, 195301 (2005).

${ }^{18}$ V. L. Malevich, Semicond. Sci. Technol. 17, 551 (2002).

${ }^{19}$ V. L. Malevich, Acta Phys. Pol. A 107, 169 (2005).

${ }^{20}$ D. Liu and J. Qin, Int. J. Infrared Millim. Waves 24, 2127 (2003).

${ }^{21}$ D. Liu and Y. Tan, Int. J. Infrared Millim. Waves 26, 1265 (2005).

${ }^{22}$ D. Liu and D. Xu, Int. J. Infrared Millim. Waves 27, 1195 (2006).

${ }^{23}$ A. Reklaitis, Phys. Rev. B 74, 165305 (2006).

${ }^{24}$ A. Reklaitis, Phys. Rev. B 77, 153309 (2008).

${ }^{25}$ A. Reklaitis, J. Appl. Phys. 108, 053102 (2010).

${ }^{26}$ A. Reklaitis, J. Appl. Phys. 109, 083108 (2011).

${ }^{27}$ D. L. Cortie and R. A. Lewis, Phys. Rev. B 84, 155328 (2011).

${ }^{28}$ C. Jacoboni and L. Reggianni, Rev. Mod. Phys. 55, 645 (1983).

${ }^{29}$ C. Jacoboni and P. Lugli, The Monte Carlo Method for Semiconductor Device Simulation (Springer-Verlag/Wien, New York, 1989).

${ }^{30}$ W. Fawcett, A. D. Boardman, and S. Swain, J. Phys. Chem. Solids 31, 1963 (1970). 